\title{
EFFECT OF RIBLETS ON NONLINEAR DISTURBANCES IN THE BOUNDARY LAYER*
}

\author{
V.G. CHERNORAI ${ }^{2}$, V.V. KOZLOV ${ }^{1}$, L.L. LOEFDAHL ${ }^{2}$, G.R. GREK ${ }^{1}$, and \\ H.H. $\mathrm{CHUN}^{3}$ \\ ${ }^{1}$ Khristianovich Institute of Theoretical and Applied Mechanics SB RAS, \\ Novosibirsk, Russia \\ ${ }^{2}$ Chalmers University of Technology, Goeteborg, Sweden \\ ${ }^{3}$ Pusan National University, Pusan, South Korea
}

(Received September 13, 2005)

\begin{abstract}
Results of experimental investigations of the nonlinear stage of sinusoidal and varicose instability of a streaky structure, which leads to multiplication of streaky structures and origination of coherent structures (such as $\Lambda$-structures), are presented. Riblets suppress the intensity of streaky structures, stabilize the flow against the development of the secondary high-frequency instability of streaky structures, and, for this reason, delay spatial turbulization of the flow. The results of these investigations can be useful for understanding the flow structure in such situations and for possible controlling of the coherent structures aimed at flow stabilization.
\end{abstract}

\section{INTRODUCTION}

It is known [1] that the laminar-turbulent transition in the case of a low degree of free-stream turbulence is associated with the development of instability waves (the socalled Tollmien - Schlichting waves). In their downstream evolution, these waves can grow linearly at first, then they experience a nonlinear stage of development, and finally lead to flow turbulization. The linear stage of development of instability waves has been studied in sufficient detail both theoretically and experimentally, but the nonlinear stage (especially its last phases) has not been adequately examined. The most important results in these studies have been achieved in physical and numerical experiments. The twodimensional Tollmien - Schlichting wave at the nonlinear stage of its evolution experiences a three-dimensional distortion when it reaches a certain amplitude, which results in emergence of typical three-dimensional $\Lambda$-structures [2,3]. The specific feature of origination and development of these structures is the fact that they are not only typical of the classical laminar-turbulent transition but are also observed in the course of evolution of more complicated flows, such as flows modulated by streamwise streaky structures of the Goertler-vortex type [4], cross-flow vortices on swept wings [1], etc., and also in the viscous sublayer of a turbulent boundary layer [5, 6]. In these situations, such structures arise, in particular, because of the secondary high-frequency instability of these flows and can manifest themselves not only as $\Lambda$-structures but also as horse-shoe ( $\Omega$-structures), hairpin, and other vortices. It is the dynamics of downstream evolution of

\footnotetext{
* This work was supported by the President of the Russian Federation (Grant No. NSh-964.2003.1) and by the Russian Foundation for Basic Research (Grant No. 05-01-00034).
}

다 V.G. Chernorai, V.V. Kozlov, L.L. Loefdahl, G.R. Grek, and H.H. Chun, 2006 
these structures that is often responsible for the transition to turbulence or to turbulence production in the viscous sublayer of a turbulent boundary layer. The initial instability of many flows is also associated with their cross-flow modulation by stationary (Goertler-type vortices, cross-flow vortices on swept wings, etc.) and nonstationary streamwise structures (streaky structures in the case of elevated free-stream turbulence, $\Lambda-, \Omega-$, and hairpin vortices, etc.). Cross-flow modulation of flows by these structures generates conditions (unstable inflectional profiles of velocity normal to the surface $\partial U / \partial y$ and across the flow $\partial U / \partial z$ ) for origination and development of secondary high-frequency oscillations whose downstream evolution leads to boundary-layer turbulization.

The high-frequency secondary instability of transitional and turbulent near-wall flows with streaky structures is often associated with the so-called sinusoidal and varicose instability. Secondary mechanisms produce different types of secondary motion: either in the form of periodic "meandering" of vortices in the cross-flow direction or in the form of horse-shoe vortices in the region of a strong cross-flow shear [7]. Such disturbances are called the sinusoidal and varicose modes, respectively. The reason for instability is an inviscid local mechanism caused by inflections in instantaneous velocity profiles both in the normal (varicose mode) and in the transverse (sinusoidal mode) directions. Both types of instability can be assumed to be important mechanisms of turbulence generation and its production in a turbulent boundary layer [8 - 10]. The sinusoidal and varicose modes of instability under controlled conditions at the linear stage and at the initial steps of nonlinear evolution were examined in [11] and, in more detail, at the late stages of nonlinear evolution in [12]. The objective of the present work is to study the possibility of controlling the nonlinear stage of development of the sinusoidal and varicose instability with the help of surface modification by riblets.

\section{EXPERIMENTAL SETUP AND MEASUREMENT PROCEDURE}

The experiments were performed under controlled conditions on a flat plate in a low-turbulent wind tunnel (Fig. 1). The streaky structure was generated by a cylindrical roughness element $1.1 \mathrm{~mm}$ high and $5.8 \mathrm{~mm}$ in diameter, which was mounted in the center of the plate at a distance $x_{0}=438 \mathrm{~mm}$ from the leading edge of the plate. The flow velocity was $U_{0}=7.8 \mathrm{~m} / \mathrm{s}$. If there were no roughness elements, the laminar boundary layer developed without any waves, and the velocity profile was close to the Blasius profile. The height of the roughness element $h=1.1 \mathrm{~mm}$ is close to the displacement thickness of the Blasius laminar boundary layer $\delta_{\mathrm{B}}^{*} \approx 1.5 \mathrm{~mm}$ for $x-x_{0}$ and $U_{0}=7.8 \mathrm{~m} / \mathrm{s}$. The Reynolds number was $\operatorname{Re}^{*} \approx \delta_{\mathrm{B}}^{*} \cdot U_{0} / v=780$ for $x=x_{0}$. If no artificial disturbances were introduced, the boundary layer with the streaky structure remained laminar within

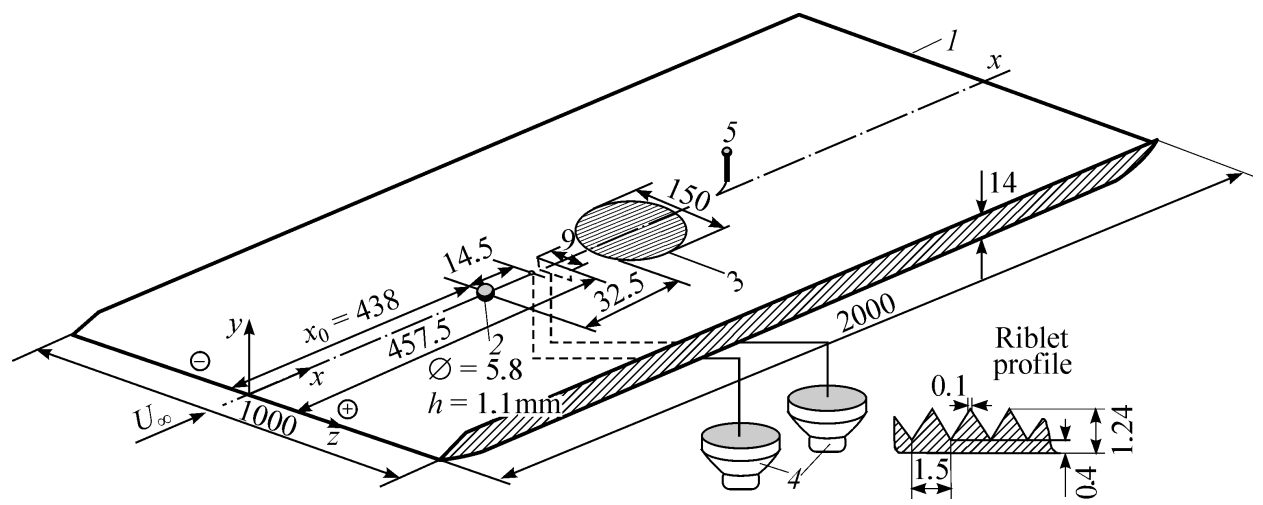

Fig. 1. Experimental setup.

1 - flat plate, 2 - roughness element, 3 - riblets, 4 - dynamic loudspeakers, 5 - hot-wire probe. 
the region where the measurements were made: $x-x_{0}=30-150 \mathrm{~mm}$. This allowed us to control the streaky-structure instability by means of artificial disturbances generated by gas injection and suction through three small orifices on the plate surface, as in [11, 12]. The excited frequency of the secondary high-frequency disturbance was $150 \mathrm{~Hz}$, which approximately corresponded to the dimensionless frequency parameter $2 \pi f v / U_{0}^{2} \cdot 10^{6}=$ $=232$. The amplitude of the secondary disturbance reached $10 \%$ of $U_{0}$ in the vicinity of the source $\left(x-x_{0}=30 \mathrm{~mm}\right)$, which allowed us to study the nonlinear stage of the process. A hot-wire anemometer was used to measure the mean streamwise component of velocity $U$ and velocity fluctuations $u_{\mathrm{rms}}$ in the space $x y z$.

\section{FLOW STRUCTURE AT THE NONLINEAR STAGE OF EVOLUTION OF SINUSOIDAL AND VARICOSE INSTABILITY OF THE STREAKY STRUCTURE}

Figure 2 shows the sinusoidal breakdown of the streaky structure. The spatial pattern of disturbance evolution (see Fig. 2,a) shows that transverse meandering of the streaky structure is observed in the beginning of breakdown, which is typical of sinusoidal instability development. Further downstream, however, the structure of the disturbed region transforms to typical coherent structures, which resemble $\Lambda$ vortices. The development of secondary disturbances is illustrated in Fig. 2, $b$. At the initial stage of evolution of this disturbance, one can observe a pair of quasi-streamwise vortices of alternating sign, which transform to $\Lambda$-structures further downstream. Thus,

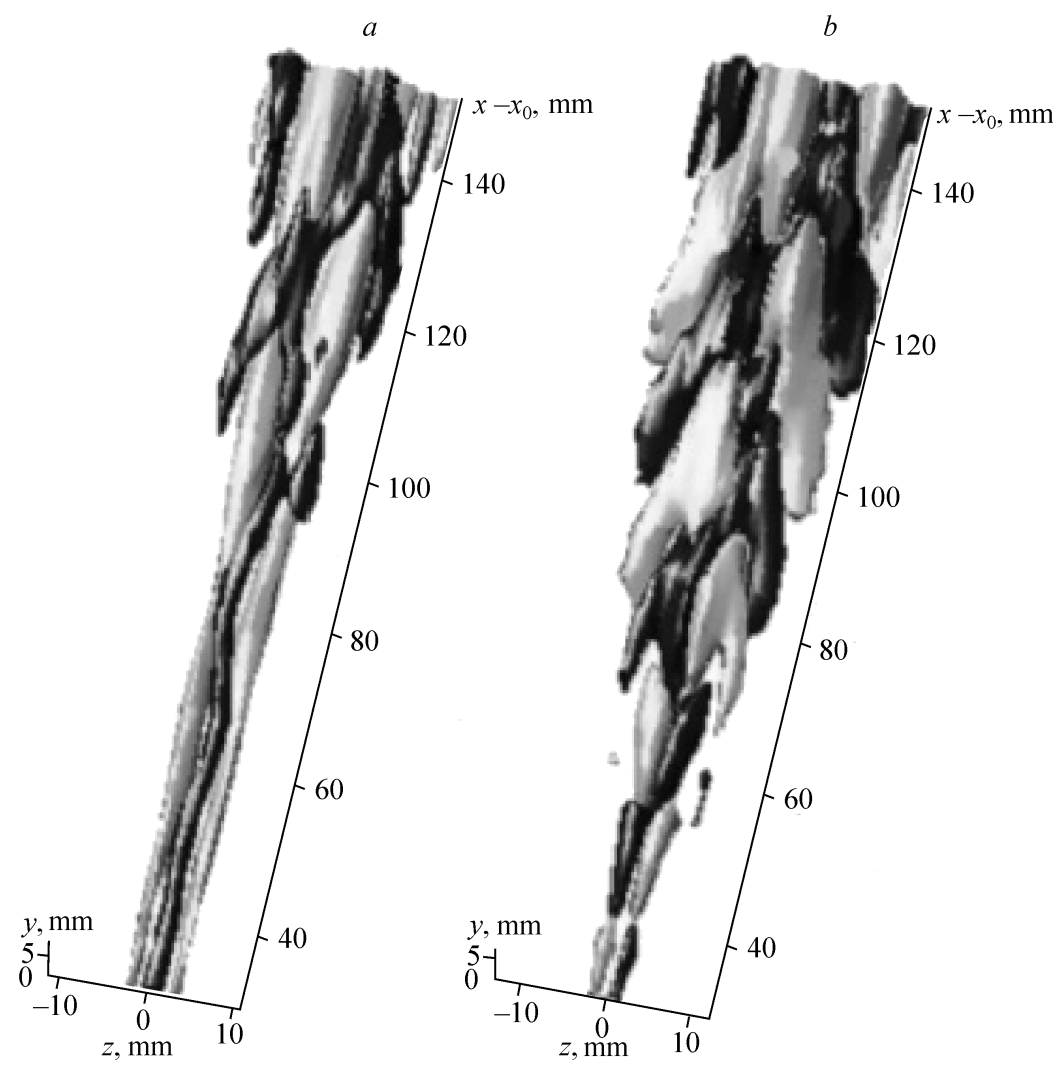

Fig. 2. Spatial patterns of sinusoidal breakdown of the streaky structure.

Effect of riblets on the mean flow parameters (levels of amplitude $U= \pm 5 \% U_{0}, a$ ) and on the fluctuating parameters of the flow (levels of amplitude $u^{\prime}= \pm 2 \% U_{0}, b$ ); the dark and light half-tones refer to velocity excess and defects, respectively. 
detailed hot-wire measurements of the nonlinear stage of sinusoidal instability development showed that the secondary high-frequency breakdown of the streaky structure is associated with the formation of $\Lambda$-structures whose downstream destruction leads to flow turbulization.

Figure 3 shows the varicose breakdown of the streaky structure. The spatial pattern of disturbance evolution (see Fig. 3,a) shows that streamwise modulation of the streaky structure by the secondary-disturbance frequency $(f=150 \mathrm{~Hz})$ is observed at the initial step, which is typical of varicose instability development. Further downstream, however, the structure of the disturbed region transforms to typical coherent structures, which resemble $\Lambda$-vortices, as in the case of sinusoidal breakdown of the streaky structure. It should be noted that these $\Lambda$-structures are asymmetric, in contrast to the previous case, i. e., the second counter-rotating vortex is at the stage of formation because of the low vorticity on transverse boundaries of the disturbed flow field. Below, in considering the evolution of the high-frequency disturbance proper, we will observe symmetric $\Lambda$-structures. Let us consider the dynamics of evolution of the secondary high-frequency disturbance generated on the streaky structure. The development of secondary disturbances is illustrated in Fig. 3, $b$. The initial stage of disturbance evolution involves a chain of quasi-streamwise vortices transformed in the downstream direction into hairpin vortices or $\Lambda$-structures. These vortices are clearly observed at $z=0 \mathrm{~mm}$ as a pair of structures of variable sign at each period of the secondary disturbance. At $z= \pm 5 \mathrm{~mm}$, the

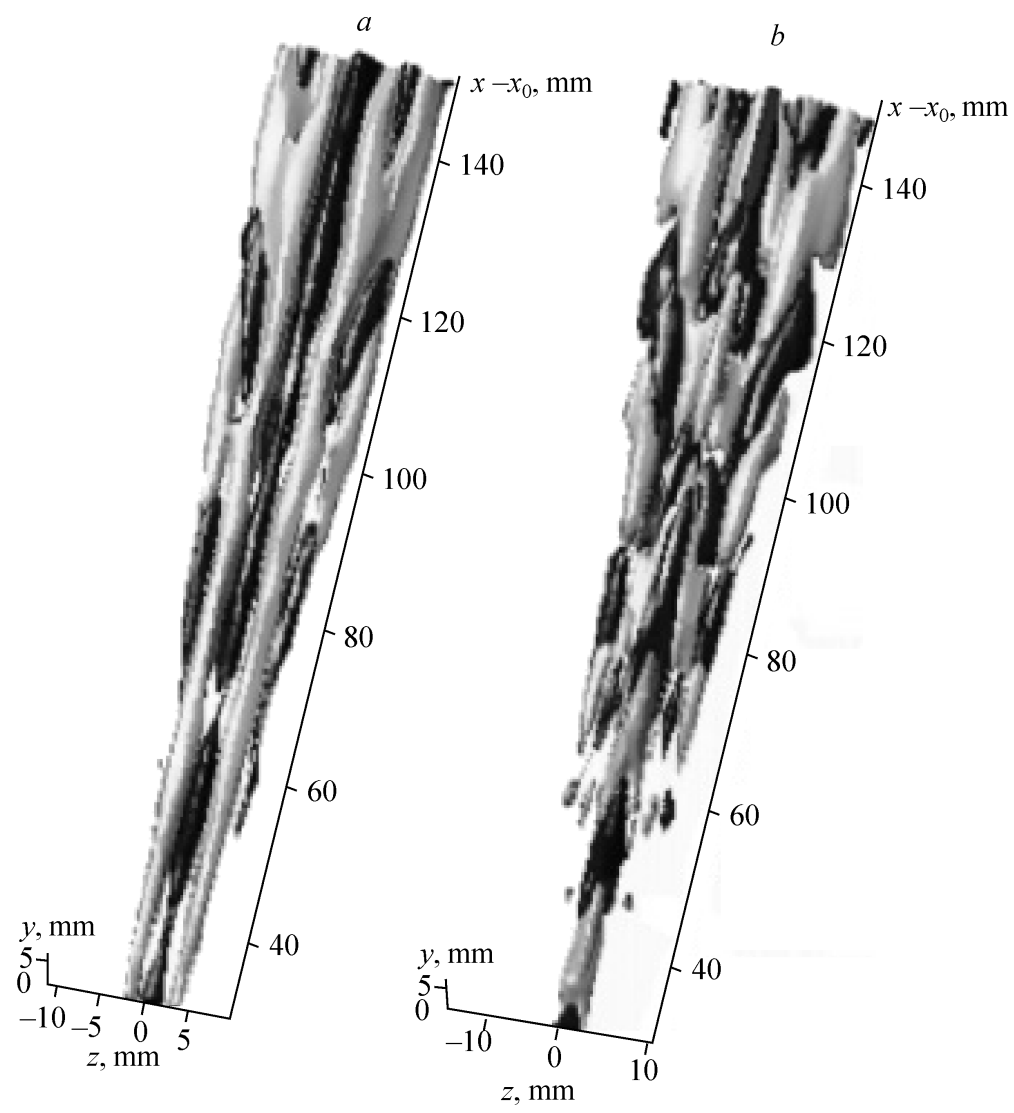

Fig. 3. Spatial patterns of varicose breakdown of the streaky structure.

Effect of riblets on the mean flow parameters (levels of amplitude $U= \pm 3 \% U_{0}, a$ ) and on the fluctuating parameters of the flow (levels of amplitude $u^{\prime}= \pm 1.5 \% U_{0}, b$ ); the dark and light half-tones refer to velocity excess and defects, respectively. 
transverse boundaries of the disturbed region, $\Lambda$-structure, or hairpin vortices become asymmetric; nevertheless, the structure of the second counter-rotating vortex of these coherent structures can be clearly seen. The study of varicose instability of a single streaky structure in the boundary layer on a swept wing [13] showed that $\Lambda$-vortices transform to asymmetric structures because of the transverse flow.

Thus, detailed hot-wire measurements of the nonlinear stage of evolution of varicose instability showed that the secondary high-frequency disintegration of the streaky structure is caused by the formation of $\Lambda$-structures, as in the case of sinusoidal breakdown of the streaky structure. The secondary instability of the streaky structure of the sinusoidal and varicose types at the nonlinear stage leads to multiplication of new streaky structures in the downstream direction, and $\Lambda$-vortices multiply in the transverse direction as the disturbance evolves further downstream. This result is important for understanding both the mechanism of turbulization of flows modulated by streaky structures and the mechanisms of turbulence production in turbulent flows where the dynamics of coherent structures of the viscous sublayer plays the determining role.

\section{CONTROL OF THE NONLINEAR STAGE OF SINUSOIDAL AND VARICOSE INSTABILITY OF THE STREAKY STRUCTURE BY MEANS OF RIBLETS}

Let us consider the possibility of controlling this instability. For this purpose, we performed experiments aimed at controlling the nonlinear stage of evolution of varicose and sinusoidal instability by applying riblets onto the surface. The stabilizing effect of riblets on the transition was noted in [14]. In the case considered, we used riblets (see Fig. 1) from the previous works $[15,16]$, which demonstrated the stabilizing effect on the transition to turbulence in a flow modulated by streamwise vortices of the Goertlertype [15] and on $\Lambda$-structures at the nonlinear stage of the classical transition with low external turbulence [16]. The riblets have a triangular profile with the peak height (or groove depth) $h=1.2 \mathrm{~mm}$, transverse size (or peak step) $s=1.5 \mathrm{~mm}$, and peak width of $0.1 \mathrm{~mm}$. The dimensionless parameter of the riblets was $s^{+}=s u^{*} / v$, where $u^{*}=$ $=\left(v|\partial u / \partial y|_{y=0}\right)^{1 / 2}$ is the laminar friction velocity and $|\partial u / \partial y|_{y=0}=0.332 U_{\infty} / \delta$ is the gradient of mean velocity on the wall $\left(\delta \sim(v X / U)^{1 / 2}\right)$. In the range of Reynolds numbers examined $\operatorname{Re}_{x}=2.63-3.25 \cdot 10^{5}$, the dimensionless transverse parameter $s^{+}$normalized to the internal variables $\left(v, u^{*}\right)$ was $20 \geq s^{+} \geq 18$, which is fairly close to a similar parameter from [16] $26 \geq s^{+} \geq 21$ and approaches the optimal parameter of riblets for a turbulent boundary layer from [17] $s^{+} \approx 15$. The effect of riblets mounted along and across the flow on flow turbulization for the sinusoidal and varicose instability of the streaky structure is illustrated in Figs. 4 and 5, respectively. The measured values of $U$ and $u_{\mathrm{rms}}$
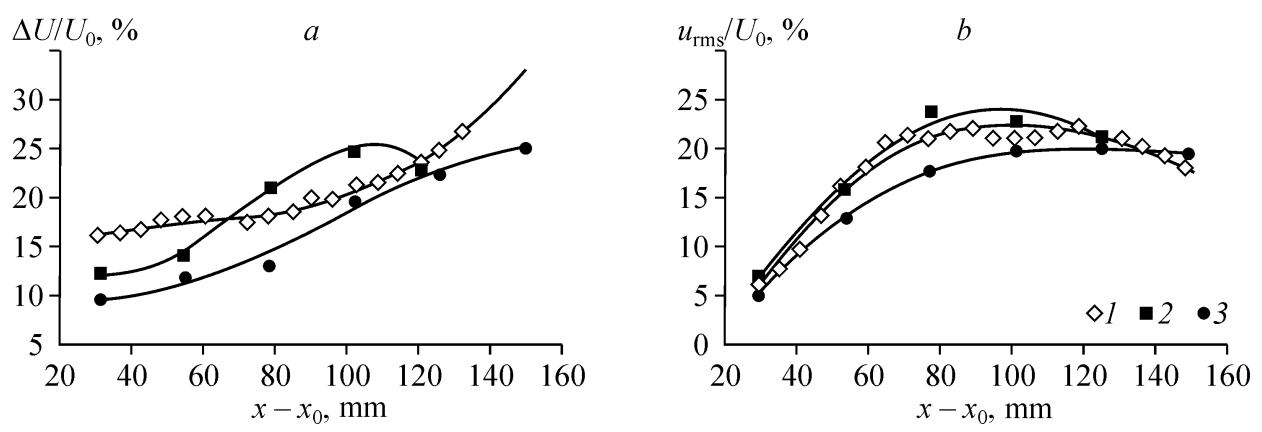

Fig. 4. Curves of downstream growth of intensities of the mean $(a)$ and fluctuating $(b)$ components of the disturbance for sinusoidal instability of the streaky structure on a smooth surface $(1)$, transversely ribbed surface (2), and streamwise ribbed surface (3). 

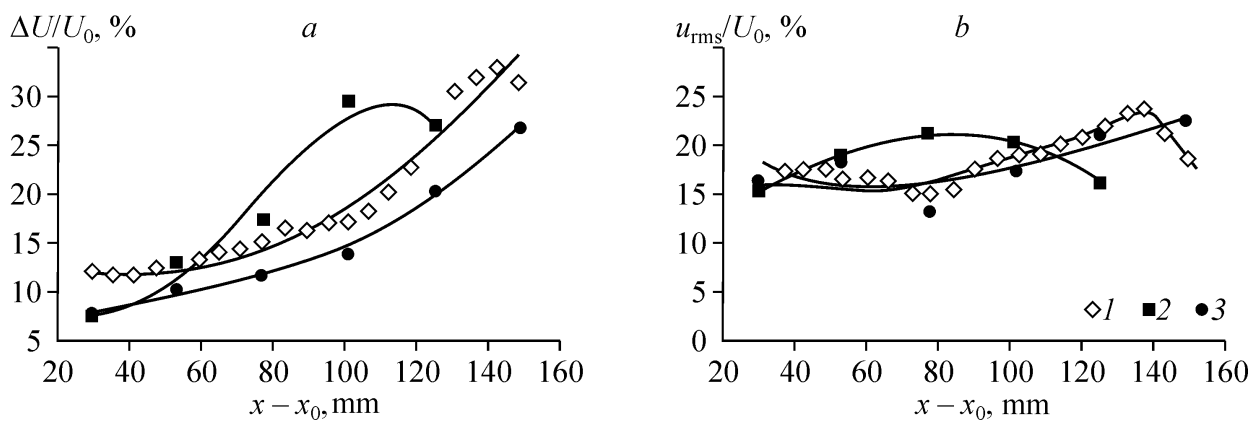

Fig. 5. Curves of downstream growth of intensities of the mean $(a)$ and fluctuating $(b)$ components of the disturbance for varicose instability of the streaky structure on a smooth surface (1), transversely ribbed surface (2), and streamwise ribbed surface (3).

for both types of positioning of the riblets are compared with these parameters measured on a smooth surface. Figures $4, a$ and 5, $a$ show the effect of riblets on the mean flow characteristics, which is presented in the form $\Delta U=1 / 2\left(\max _{y, z}\left\{U^{\prime}\right\}-\min _{y, z}\left\{U^{\prime}\right\}\right)$, where $\left[\max _{y, z}\left\{U^{\prime}\right\}\right]$ is the maximum velocity excess in the $y, z$ plane and $\left[\min _{y, z}\left\{U^{\prime}\right\}\right]$ is the minimum velocity defect in the same plane. The evolution of velocity fluctuations is shown in Figs. $4, b$ and $5, b$; it is characterized by the maximum value of root-meansquare fluctuations of velocity $\left[u_{\mathrm{rms}}=\max _{y, z}\left\{u_{\mathrm{rms}}\right\}\right]$ in the $y, z$ plane. The effect of riblets on the mean parameters of the flow, i. e., directly on intensity of streaky structures, shows that this parameter is significantly lower within the entire range of measurements in the downstream direction if the model has riblets in the streamwise direction, as compared to a smooth surface, both for sinusoidal (see Fig. 4, $a$ ) and for varicose instability (see Fig. 5, a). Transverse ribbing leads to a drastic increase in intensity of the structure and flow turbulization further downstream, which is evidenced by the change in the sign of the derivative $\partial U / \partial x$ at $x \approx 100 \mathrm{~mm}$ in Fig. 4, $a$ and $x \approx 110 \mathrm{~mm}$ in Fig. 5, $a$. If we consider the growth curves of the secondary high-frequency disturbance on the ribbed and smooth surfaces, we can note a drastic increase in its intensity (from $5 \% U_{0}$ at $x-x_{0}=30 \mathrm{~mm}$ to approximately $20 \% U_{0}$ at $x-x_{0}=90 \mathrm{~mm}$ ) at the initial stage of sinusoidal instability development (see Fig. $4, b$ ) and an approximately identical level of intensity $\left(15-20 \% U_{0}\right.$ in the same spatial interval) for varicose instability (see Fig. 5, b). This fact confirms the known conclusion [18] that it is because of the drastic increase in disturbance amplitude, which leads to rapid turbulization of the flow, that sinusoidal instability is more dangerous than varicose instability. Therefore, to compare the influence of riblets on both types of instability in one spatial interval, the initial amplitudes of the secondary disturbance had different values; nevertheless, the spatial coordinate where the disturbance amplitude reached approximately an identical level (about $20 \% U_{0}$ ) was the same $\left(x-x_{0} \approx 80 \mathrm{~mm}\right)$ in both cases. It should be noted that the first measurement $\left(x-x_{0}=30 \mathrm{~mm}\right)$ was made outside the ribbed surface. The point of flow transition to a turbulent state is normally associated with the point where the disturbance-growth curve reaches a maximum and starts to fall down. As is seen in the figures (see Figs. 4, $b$ and $5, b$ ), no transition to turbulence is observed in the flow with downstream-aligned riblets. In the case of sinusoidal instability, the disturbance amplitude becomes saturated, but its growth curve practically does not go down; in the case of varicose instability, the disturbance amplitude continues to grow. The flow on a smooth surface displays the transition to turbulence at $x-x_{0} \approx 120 \mathrm{~mm}$ (sinusoidal instability, see Fig. 4, $b$ ) and at $x-x_{0} \approx$ $\approx 140 \mathrm{~mm}$ (varicose instability, see Fig. 5, $b$ ). If the riblets are aligned in the transverse direction, the transition point is shifted upstream approximately by 30 and 
$50 \mathrm{~mm}$ as compared to the smooth surface in the case of sinusoidal and varicose instability, respectively (see Figs. $4, b$ and $5, b$ ). It should also be noted that additional investigations were performed to study the influence of a step generated by riblets themselves on the measurement results. In these investigations, the riblets were replaced by a smooth insert of the same size. No effect of such a step was observed, which agrees with the data of [16].

Thus, the experimental investigations in the field of controlling the development of sinusoidal and varicose instability of the streaky structure at the nonlinear stage of its evolution showed that streamwise ribbing of the surface stabilizes the mean flow, i. e., reduces the intensity of streaky structures. In turn, this favors a decrease in the amplitude of velocity fluctuations, i. e., a decrease in intensity of secondary high-frequency disturbances developed on the streaky structures. Finally, this prevents the flow transition to a turbulent state, whereas the flow on a smooth surface experiences the transition in this spatial interval. Transverse ribbing of the surface destabilizes the mean flow, i. e., increases the intensity of streaky structures, which, in turn, favors the increase in the amplitude of velocity fluctuations, i. e., intensity of secondary high-frequency disturbances developed on these structures. Finally, this leads to faster turbulization of the flow in space, as compared to the case on a smooth surface.

\section{CONCLUSIONS}

Experimental investigations of the nonlinear stage of development of sinusoidal and varicose instability of the streaky structure and the possibility of controlling this process by means of riblets allowed the following conclusions.

- It is shown that the secondary high-frequency instability of the streaky structure at the nonlinear stage leads to multiplication of streaky structures and to origination and development of coherent structures, such as $\Lambda$-structures, in the case of both sinusoidal and varicose instability.

- The stabilizing effect of streamwise-aligned riblets on both types of instability is found; this effect is characterized by a decrease in intensity of streaky structures and secondary high-frequency disturbances and by an overall delay of flow turbulization.

- The destabilizing effect of transversely aligned riblets on both types of instability is demonstrated; this effect is characterized by an increase in intensity of streaky structures and secondary high-frequency disturbances and by faster turbulization of the flow.

\section{REFERENCES}

1. A.V. Boiko, G.R. Grek, A.V. Dovgal, and V.V. Kozlov, The Origin of Turbulence in Near-Wall Flows, Springer-Verlag, Berlin et al., 2002, P. 1-263.

2. P.S. Klebanoff, K.D. Tidstrom, and L.M. Sargent, The three-dimensional nature of boundary-layer instability, J. Fluid Mech., 1962, Vol. 12(1), P. 1-34.

3. W.S. Saric, V.V. Kozlov, and V.Ya. Levchenko, Forced and unforced subharmonic resonance in boundary layer transition, AIAA Paper No. 84-0007, 1984.

4. J.M. Floryan, On the Goertler instability of boundary layers, Technical Report of National Aerospace Laboratory, TR-1120, 1991, P. 1-45.

5. J. Zhou, R.J. Adrian, S. Balachandar, and T.M. Kendall, Mechanisms for generating coherent packets of hairpin vortices in channel flow, J. Fluid Mech., 1999, Vol. 387, P. 353-396.

6. R.J. Adrian, C.D. Meinhart, and C.D. Tomkins, Vortex organization in the outer region of the turbulent boundary layer, Ibid., 2000, Vol. 422, P. 1-23.

7. F. Li and M.R. Malik, Fundamental and subharmonic secondary instabilities of Goertler vortices, Ibid., 1995, Vol. 82, P. 255-290.

8. L. Brandt and D.S. Henningson, Transition of streamwise streaks in zero-pressure-gradient boundary layers, Ibid., 2002, Vol. 472, P. 229-261.

9. J.D. Swearingen and R.F. Blackwelder, The growth and breakdown of streamwise vortices in the presence of a wall, Ibid., 1987, Vol. 182, P. 225-290.

10. M. Skote, J.H. Haritonidis, and D.S. Henningson, Varicose instabilities in turbulent boundary layers, Phys. Fluids, 2002, Vol. 4, No. 7, P. 2309-2323. 
11. M. Asai, M. Minagawa, and M. Nishioka, The stability and breakdown of near-wall low-speed streak, J. Fluid Mech., 2002, Vol. 455, P. 289-314.

12. Yu.A. Litvinenko, V.G. Chernorai, V.V. Kozlov, L. Loefdahl, G.R. Grek, and H. Chun, Nonlinear sinusoidal and varicose instability in the boundary layer (Review), Thermophysics and Aeromechanics, 2004, Vol. 11, No. 3, P. 329-353.

13. Yu.A. Litvinenko, G.R. Grek, V.V. Kozlov, L. Loefdahl, and V.G. Chernorai, Experimental investigation of a streaky structure varicose instability in a swept wing boundary layer, Ibid., 2004, Vol. 11, No. 1, P. 13-21.

14. P. Luchini, F. Manzo, and A. Pozzi, Resistance of a grooved surfaces to parallel and crossflow, J. Fluid Mech., 1991, Vol. 228, P. 87-109.

15. G.R. Grek, V.V. Kozlov, B.G.B. Klingmann, and S.V. Titarenko, The influence of riblets on a boundary layer with embedded streamwise vortices, Phys. Fluids, 1995, Vol. 7, No. 10, P. 2504-2506.

16. G.R. Grek, V.V. Kozlov, and S.V. Titarenko, An experimental study of the influence of riblets on transition, J. Fluid Mech., 1996, Vol. 315, P. 31-49.

17. M.J. Walsh, Drag characteristics of V-groove and transverse curvature riblets, in: Viscose Drag Reduction, G.R. Houhg (Ed.), Washington, DC, AIAA, 1980, P. 168-184.

18. P. Andersson, L. Brandt, A. Bottaro, and D.S. Henningson, On the breakdown of boundary layers streaks, J. Fluid Mech., 2001, Vol. 428, P. 29-60. 